\title{
Retrospective Database Analysis of Treatment Patterns Among Patients with Pulmonary Arterial Hypertension
}

\author{
Sean Studer - Michael Hull · Janis Pruett - Caitlin Elliott · \\ Yuen Tsang · William Drake
}

Received: October 21, 2019 / Published online: December 19, 2019

(C) The Author(s) 2019

\begin{abstract}
Introduction: Release of the 2015 European Society of Cardiology (ESC)/European Respiratory Society (ERS) guidelines put increased emphasis on using combination therapy, either as upfront or sequential therapy among patients with pulmonary arterial hypertension (PAH). However, with these recommendations and the therapy advances made in the last several years, little is known on the real-world treatment patterns among patients with $\mathrm{PAH}$, particularly before and after publication of the 2015 ESC/ERS guidelines. Methods: This was a retrospective study of adult commercial and Medicare Advantage with Part D (MAPD) enrollees with at least one claim for a PAH-related medication from January 01, 2012 to March 31, 2017, at least one medical claim with a pulmonary hypertension diagnosis, and
\end{abstract}

Enhanced digital features To view enhanced digital features for this article go to https://doi.org/10.6084/ m9.figshare.11317694.

S. Studer

NYC Health + Hospitals/Kings County, New York, NY, USA

M. Hull $(\bowtie) \cdot$ C. Elliott

Optum, Eden Prairie, MN, USA

e-mail: michael.hull@optum.com

J. Pruett $\cdot$ Y. Tsang $\cdot$ W. Drake

Actelion Pharmaceuticals US, Inc, South San

Francisco, CA, USA continuous health plan enrollment at least 6 months prior to and at least 12 months following the date of the first pharmacy claim for PAH-related therapy (index date). Patients were divided into cohorts based on prescription of monotherapy or combination therapy and index date category (2012-2013, January 2014-July 2015, and August 2015-March 2017). Results: Out of 1878 patients, 90.8\% initiated with monotherapy and $9.2 \%$ initiated with combination therapy. The percentage of patients with index combination therapy increased from $5.7 \%$ in $2012-2013$ to $13.0 \%$ in August 2015-March 2017. Patients with index combination therapy had better persistence (11.6 months versus 10.3 months) and adherence $(0.95$ versus 0.85$)$. Overall, the discontinuation rate was $40 \%$ and was higher in monotherapy versus combination therapy patients (42.8\% versus $12.2 \%$ ). Approximately $30.2 \%$ of patients had a second regimen, of which 50\% were combination regimens. The time to combination therapy initiation decreased from 10.5 months in 2012-2013 to 3.4 months in August 2015-March 2017.

Conclusions: The majority of patients initiated monotherapy treatment for PAH, most often a phosphodiesterase 5 inhibitor (PDE5i). Patients with upfront combination therapy increased following publication of the 2015 ESC/ERS guidelines, indicating that physicians responded to the guideline's option of prescribing upfront combination therapy. 
Keywords: Adherence; Combination therapy; Discontinuation; Monotherapy; PAH; Pulmonary arterial hypertension; Switch; Treatment patterns

\section{Key Summary Points}

\section{Why carry out this study?}

Several treatment advances have been made for patients with pulmonary arterial hypertension (PAH); however, little is known about the real-world treatment patterns, particularly with respect to treatment patterns before and after the publication of the $2015 \mathrm{ESC} / \mathrm{ERS}$ guidelines

As new treatments and advances are introduced and the effects of combination therapies are compared against those of current prostacyclin formulations, the medical community is interested in understanding therapy patterns and outcomes of patients prescribed combination therapy compared to monotherapy as it advocates for further cost-effective treatments

The purpose of this study is to examine medication treatment patterns among patients with PAH who were newly initiating therapy

\section{What was learned from the study?}

Patients with upfront combination therapy increased and the time to sequential combination therapy decreased following publication of the $2015 \mathrm{ESC} /$ ERS guidelines, indicating that physicians were prescribing according to recommended guidelines

Patients who initiated combination therapy had greater adherence and persistence with fewer discontinuations than patients who initiated monotherapy

The majority of patients initiated monotherapy treatment for $\mathrm{PAH}$, most often PDE5is, despite market availability of several new medications

\section{INTRODUCTION}

Pulmonary arterial hypertension (PAH) is a progressive, potentially life-threatening disease characterized by high pulmonary arterial blood pressure and increased pulmonary vascular resistance as a result of narrowing of the pulmonary arteries. If left untreated, PAH can result in right heart failure and death. Prevalence estimates of $\mathrm{PAH}$ range from 10 to 26 cases per million population, with $2-8$ new cases per million annually $[1,2]$.

Several medications indicated for $\mathrm{PAH}$ have been approved in the USA since 2013, including the soluble guanylyl cyclase (sGC) stimulator riociguat, the oral prostacyclin analogue treprostinil, the oral endothelin receptor antagonist (ERA) macitentan, the selective agonist of the prostacyclin IP receptor selexipag, and an indication for the combination of ambrisentan plus tadalafil.

Combination therapy offers the potential to target multiple pathways simultaneously, potentially improving outcomes and lowering dose requirements. Several clinical trials, including SERAPHIN and GRIPHON, have noted reductions in the risk of clinical worsening [3-8] and long-term morbidity/mortality [9-11] among patients on combination therapy. In the AMBITION treatment strategy trial, initial combination therapy with an ERA (ambrisentan) plus a phosphodiesterase 5 inhibitor (PDE5i, tadalafil) was compared to pooled monotherapy in treatment-naïve patients with $\mathrm{PAH}$ [7]. Investigators noted a decreased risk of clinical failure events, reduced risk of morbidity and mortality, and improved functional class, exercise capacity, and hemodynamics among patients on the combination of ambrisentan plus tadalafil versus monotherapy.

The European Society of Cardiology (ESC)/ European Respiratory Society (ERS) issued a guideline update in 2015 stating that initial treatment strategy should be guided by individual risk assessment levels [12]. The recommended treatment algorithm was recently upheld at the 6th World Symposium on Pulmonary Hypertension [13]. Owing to the documented benefits, some suggest that patients 
initiate combination therapy preemptively, compared to the historical approach that recommended adding to oral monotherapy in response to clinical worsening. In recognition of results from the AMBITION trial [7], the new guidelines now list upfront combination therapy with ambrisentan and tadalafil as a class I recommendation for patients with functional class II and III PAH [12]. Equal to the recommendation of upfront combination therapy, stepwise combination therapy with macitentan added to sildenafil, riociguat added to bosentan, selexipag added to an ERA or PDE5i, or sildenafil added to epoprostenol (for functional class III) are also recommended. In patients with inadequate clinical response to monotherapy or dual therapy, an additional agent targeting the prostacyclin $\left(\mathrm{PGI}_{2}\right)$ pathway should be considered. Medications targeting the $\mathrm{PGI}_{2}$ pathway were previously only used for patients with severe disease given the complex routes of administration and the potential for side effects; however, the advent of oral $\mathrm{PGI}_{2}$-targeted therapies has opened the option for earlier use of combination therapy. As a result of the progressive nature of $\mathrm{PAH}$, some argue that triple combination therapy that targets all three pathogenic pathways should be started immediately upon diagnosis to delay pulmonary vascular remodeling [14].

Results from several real-world studies have raised concerns that physicians may not be prescribing according to evidence-based guidelines and consensus recommendations [15-18]. In the RePHerral study, $61 \%$ of patients presented with advanced $\mathrm{PAH}$, but only $30 \%$ were on PAH-specific medications [16]. Among patients who received medication, $57 \%$ were not prescribed according to published guidelines upon referral to a specialty center. In REVEAL registry participants, only $56 \%$ of patients with a PAH-related death were treated with intravenous prostacyclin analogues at the time of death despite the recommendation by the American Heart Association/American College of Cardiology guidelines for intravenous prostacyclin analogue therapy among patients with severe disease [19].

In spite of the treatment advances made, little is known about the real-world treatment patterns among patients with $\mathrm{PAH}$, particularly with respect to treatment patterns before and after the publication of the 2015 ESC/ERS guidelines. As new treatments and advances are introduced and the effects of combination therapies are compared against those of current prostacyclin formulations, the medical community is interested in understanding therapy patterns and outcomes of patients prescribed combination therapy compared to monotherapy as it advocates for further cost-effective treatments. The purpose of this study is to examine medication treatment patterns among patients with PAH newly initiating therapy. This study serves to update a previous study [20], lengthening the time to specifically compare treatment patterns among time periods prior to and following publication of the 2015 ESC/ERS treatment guidelines for pulmonary hypertension.

\section{METHODS}

This was a retrospective database study using medical and pharmacy claims and enrollment information from July 01, 2011 to March 31, 2018 (study period). Included patients were commercial and Medicare Advantage with Part $\mathrm{D}$ health plan members in the Optum Research Database (ORD), a large, de-identified, nationally representative, and diverse database. Medical claims were coded using the International Classification of Diseases, Ninth and Tenth Revisions, Clinical Modification (ICD-9-CM and ICD-10-CM) diagnosis and procedure codes, Healthcare Common Procedure Coding System (HCPCS) codes, and place of service codes. Outpatient pharmacy claims were coded using National Drug Codes for dispensed medication, including both the quantity dispensed and the dose and days' supply. This study did not require the use of identifiable protected health information; thus, institutional board approval or waiver of authorization was not required.

\section{Study Population}

To be included in the analysis, patients must have had at least one claim for a PAH-related 
medication from January 01, 2012 to March 31, 2017 with the date of the first pharmacy claim considered as the index date. Patients were also required to have at least one medical claim with a diagnosis for pulmonary hypertension (ICD-9CM codes 416.0, 416.8, or 416.9; ICD-10-CM codes I27.0, I27.2, I27.20, I27.21, I27.29, I27.81, I27.89, I27.9) in any position in the 6-month period prior to and including the index date. Additional inclusion criteria included continuous enrollment in the health plan with medical and pharmacy benefits for at least 6 months prior to (baseline period) and at least 12 months including and following the index date (follow-up period) and aged at least 18 years as of the index year. Patients who had a pharmacy claim for a PAH-related medication during the 6-month baseline period or those with unlisted gender, age, geographic region, or insurance plan type were excluded from the study.

\section{Cohorts}

Cohorts were selected on the basis of the prescription regimen filled during the first 30 days following the index date. Cohorts included the monotherapy cohort (pharmacy claim for only one PAH-related medication class) and the combination therapy cohort (pharmacy claims for more than one PAH-related medication class). Patients were further categorized into the following index date categories: 2012-2013, January 2014-July 2015, and August 2015-March 2017. The date categories were chosen in part around the publication of the AMBITION study (August 2015) and the ESC/ ERS guidelines (September 2015) to show treatment patterns immediately prior to and immediately following publication.

\section{Study Measures}

\section{Baseline Patient Demographic and Clinical Characteristics}

Baseline demographic and clinical characteristics collected included age, sex, insurance type, pharmacy coverage, geographic region, Charlson comorbidity score $[21,22]$, and comorbidities (identified from diagnosis codes and the Clinical Classifications Software managed by the Agency for Healthcare Research [AHRQ] based on ICD-9-CM and ICD-10-CM codes) [23].

\section{Treatment Patterns}

Treatment patterns were assessed during the 12-month follow-up period. The first PAH-related treatment medications filled in the 30 days including and following the index date were considered the index medication regimen. Whether patients discontinued (gap in therapy at least 90 days after the run-out of days' supply of the last prescription filled prior to the gap in therapy), augmented (addition or fill or a new PAH-related medication prior to discontinuation of the ongoing regimen), or switched (discontinuation of a PAH-related medication and fill for a new PAH-related medication) their index regimen was recorded. Persistence, defined as the months from the start of the first fill until therapy discontinuation or switch was also measured. Adherence to the index regimen was assessed using the proportion of days covered (PDC) calculated by division of the number of days of available medication by the number of days between the first prescription claim through the end of the study period [24]. Among patients on combination therapy, PDC was based on possession of any medication in the regimen, as adherence for individual components of related medications tends to correlate closely [25]. PDC was corrected for inpatient events under the assumption that medication was supplied by the facility during the stay. A threshold above $80 \%$ was considered highly adherent. Drug class was recorded for patients initiating a second or third PAH medication regimen and the time to combination therapy was calculated for those initiating a combination regimen.

\section{Analysis}

Numbers and percentages were provided for dichotomous and polychotomous variables, and means and standard deviations were calculated for continuous variables. Kaplan-Meier 
analyses were performed to show the time to discontinuation, augmentation, and switch of the index PAH medication regimen. Statistical analyses were performed using SAS 9.4 (SAS Institute, Cary, NC, USA).

\section{RESULTS}

\section{Baseline Demographic and Clinical Characteristics}

A total of 1878 patients were included in the study analysis (Fig. 1). Patients averaged $2.4 \pm 1.3$ years of follow-up (Table 1 ). The mean age was $66.4 \pm 13.3$ years and the majority were female $(64.4 \%)$ and enrolled in a MAPD health plan $(64.9 \%)$. Patients prescribed monotherapy at index tended to be older and were more likely to be enrolled in a MAPD plan or be male than patients prescribed combination therapy at index. The mean Charlson comorbidity score was $3.3 \pm 2.0$ and $67.4 \%$ of patients had a score of at least 3. Notable comorbidities in this population included hypertension (81.7\%), type 2 diabetes (40.9\%), sleep apnea (38.3\%), obesity $(28.8 \%)$, thyroid disease $(24.7 \%)$, and depression $(15.8 \%)$.

\section{Treatment Patterns}

A total of 1706 patients $(90.8 \%)$ initiated monotherapy and 172 patients (9.2\%) initiated combination therapy as their index regimen (Table 1). An index regimen of PDE5i, ERA, or prostacyclin monotherapy was prescribed in $64.1 \%, 16.7 \%$, and $6.1 \%$ of all study patients, respectively (Fig. 2a). Among patients who initiated combination therapy as their index regimen, the majority had a PDE5i $(86.1 \%)$ or an ERA (78.5\%) among their regimens. The percentage of patients who had a PDE5i among their combination regimen remained stable over the time periods (data not shown). The percentage of patients prescribed an ERA as part of their combination regimen increased from $64.9 \%$ in $2012-2013$ to $86.5 \%$ in August 2015-March 2017. The most commonly prescribed combination regimen among all time periods was an ERA plus PDE5i, increasing from $48.7 \%$ to $70.8 \%$ of index combination regimens from 2012-2013 to August 2015-March 2017, respectively. Patients prescribed a prostacyclin as part of their index combination therapy decreased from $51.4 \%$ in $2012-2013$ to $20.8 \%$ in August 2015-March 2017. Prescriptions for a PDE5i plus prostacyclin decreased from 35.1\%

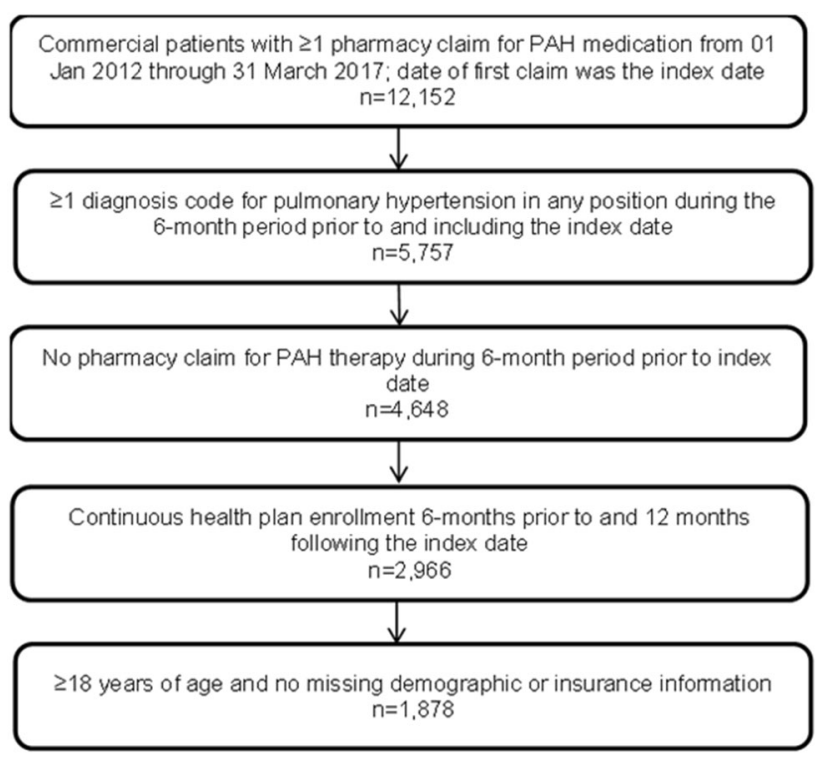

Fig. 1 Patient sample selection. PAH pulmonary arterial hypertension 
Table 1 Baseline demographic and clinical characteristics

\begin{tabular}{|c|c|c|c|c|}
\hline \multirow[t]{2}{*}{ Demographic characteristics } & \multirow{2}{*}{$\begin{array}{l}\text { Total } \\
(N=1878)\end{array}$} & \multicolumn{2}{|c|}{ Index medication therapy } & \multirow[t]{2}{*}{$p$ value } \\
\hline & & $\begin{array}{l}\text { Monotherapy } \\
(N=1706)\end{array}$ & $\begin{array}{l}\text { Combination } \\
\text { therapy } \\
(N=172)\end{array}$ & \\
\hline Age, mean (SD) & $66.4(13.3)$ & $66.9(13.1)$ & $61.8(14.0)$ & $<0.001$ \\
\hline Follow-up duration (years), mean (SD) & $2.4(1.3)$ & $2.5(1.3)$ & $2.3(1.2)$ & 0.099 \\
\hline Female gender, $n(\%)$ & $1209(64.4)$ & $1079(63.3)$ & $130(75.6)$ & 0.001 \\
\hline \multicolumn{5}{|l|}{ Insurance type, $n(\%)$} \\
\hline Commercial & $660(35.1)$ & $585(34.3)$ & $75(43.6)$ & 0.015 \\
\hline Medicare & $1218(64.9)$ & $1121(65.7)$ & $97(56.4)$ & 0.015 \\
\hline \multicolumn{5}{|l|}{ US Census Region, $n(\%)$} \\
\hline Northeast & $230(12.3)$ & $214(12.5)$ & $16(9.3)$ & 0.216 \\
\hline Midwest & $547(29.1)$ & $498(29.2)$ & $49(28.5)$ & 0.847 \\
\hline South & $825(43.9)$ & $746(43.7)$ & $79(45.9)$ & 0.579 \\
\hline West & $276(14.7)$ & $248(14.5)$ & $28(16.3)$ & 0.539 \\
\hline $\begin{array}{l}\text { Baseline Charlson comorbidity score } \\
\text { (continuous), mean (SD) }\end{array}$ & $3.3(2.0)$ & $3.4(2.0)$ & $3.1(1.9)$ & 0.137 \\
\hline \multicolumn{5}{|l|}{ Common comorbidities, $n(\%)$} \\
\hline Systemic hypertension & $1535(81.7)$ & $1399(82.0)$ & $136(79.1)$ & 0.342 \\
\hline Chronic obstructive pulmonary disease & $881(46.9)$ & $808(47.4)$ & $73(42.4)$ & 0.218 \\
\hline Type 2 diabetes & $768(40.9)$ & $713(41.8)$ & $55(32.0)$ & 0.013 \\
\hline Sleep apnea & $719(38.3)$ & $662(38.8)$ & $57(33.1)$ & 0.145 \\
\hline Obesity & $541(28.8)$ & $493(28.9)$ & $48(27.9)$ & 0.784 \\
\hline Thyroid disease & $464(24.7)$ & $421(24.7)$ & $43(25.0)$ & 0.926 \\
\hline Depression & $296(15.8)$ & $269(15.8)$ & $27(15.7)$ & 0.981 \\
\hline
\end{tabular}

of all combination regimens in 2012-2013 to $12.5 \%$ of all combination regimens in August 2015-March 2017. Approximately 37.8\% of patients remained on their index regimen until the end of the study period and $32.0 \%$ discontinued without a second regimen.

The mean PDC for all regimens over the study period was $0.86 \pm 0.22$ (Fig. 3). PDC remained stable over the different time periods for patients on both monotherapy and combination therapy. Patients prescribed combination therapy at index had a higher mean PDC than patients on monotherapy $(0.95 \pm 0.13$ versus $0.85 \pm 0.23)$. More than three-fourths of patients $(76.1 \%)$ had a PDC of at least $80 \%$.

Approximately $40.0 \%$ of patients discontinued their index medication (data not shown). Among patients who discontinued their index regimen, $80.3 \%$ did so within 1 year of the index date and $80.0 \%$ did not initiate a second regimen. The percentage of patients enrolled in 2012-2013 who discontinued was slightly higher than the percentage of patients enrolled in 2014-March 2017 who discontinued (44.1\% 

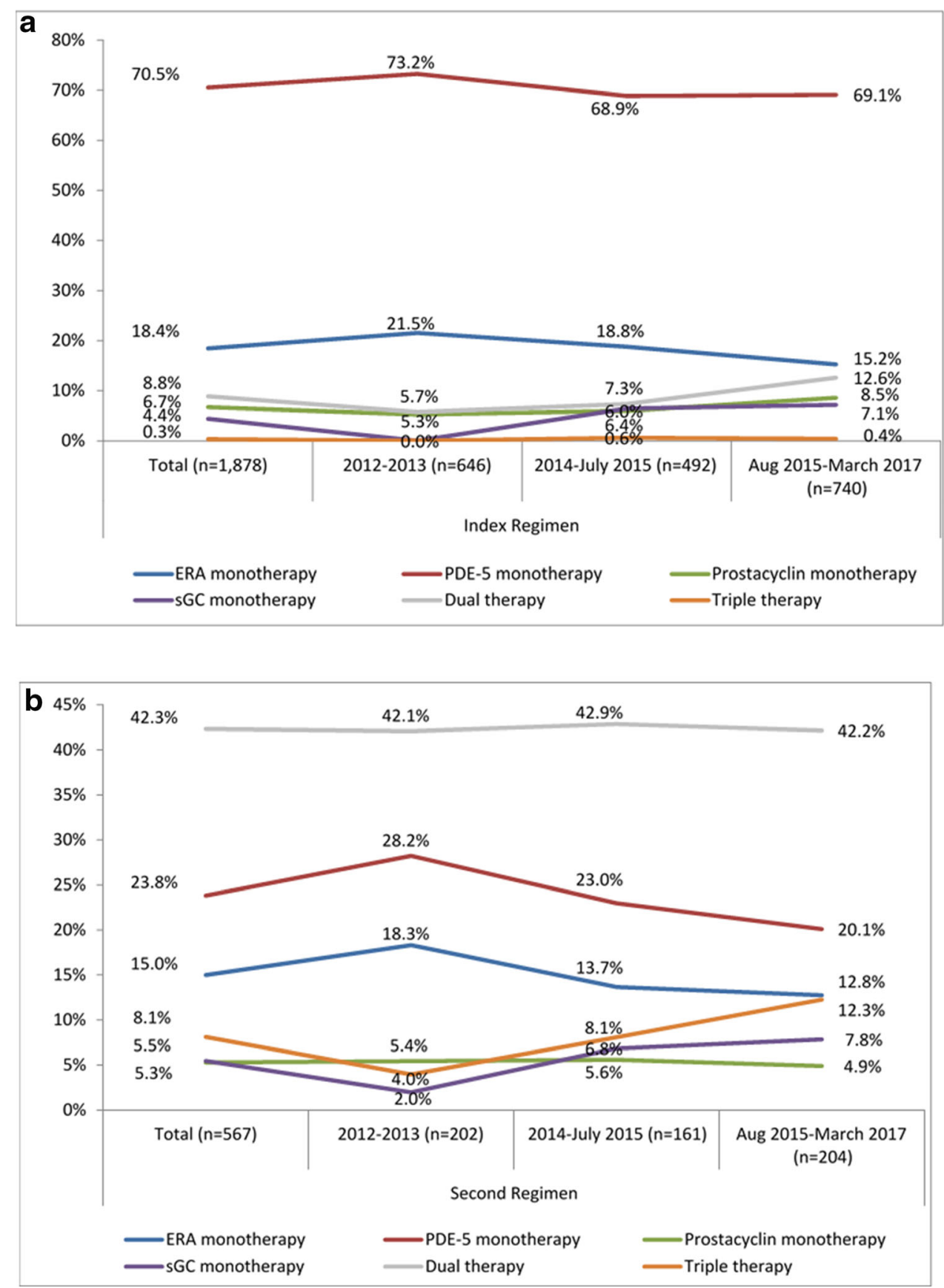

Fig. 2 PAH medication regimens. a Index regimen, b Second regimen. ERA endothelin receptor antagonist, PDE-5 phosphodiesterase 5 inhibitor, sGC soluble guanylyl cyclase

versus $37.8 \%$ ). Patients initiated on monotherapy were more likely to discontinue their medication than patients initiated on combination therapy (42.8\% versus $12.2 \%)$. The probability of discontinuing the index regimen within 1 year was $32.1 \%$ (Fig. 4). This percentage was lower among patients with an index combination regimen $(6.4 \%)$ than among patients with a monotherapy regimen (34.7\%).

The probability of augmentation of the index regimen within 1 year of the index date was $10.5 \%$ (Fig. 5). This percentage was slightly 


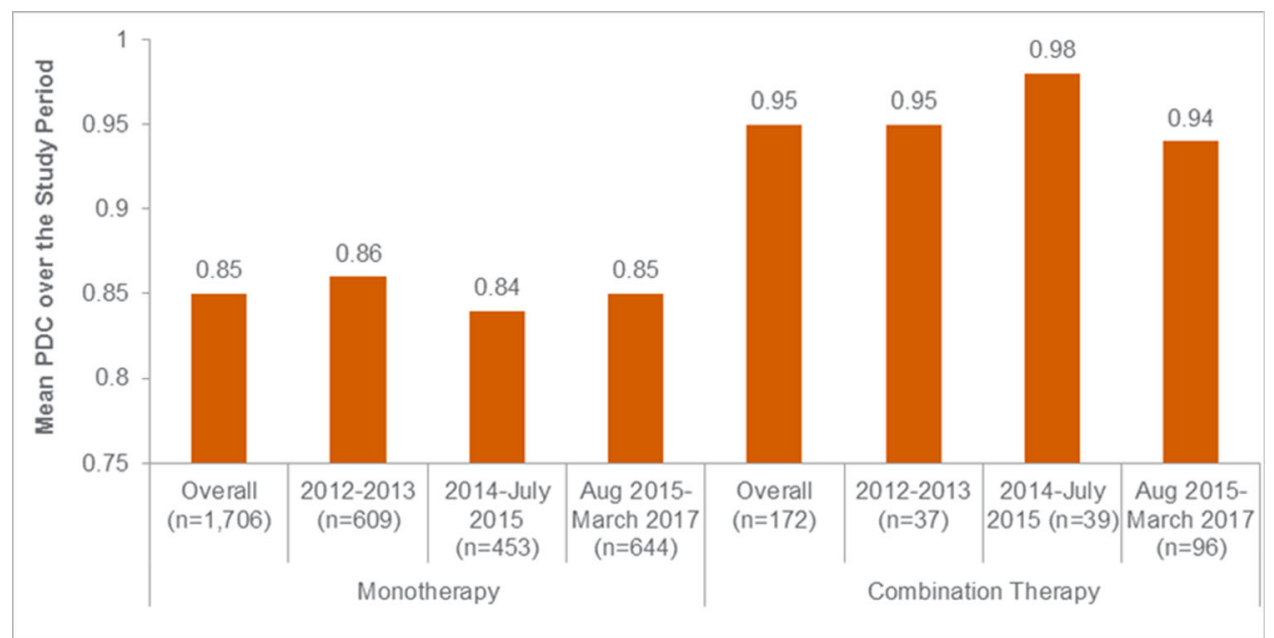

Fig. 3 Mean PDC for all regimens over the study period. PDC proportion of days covered

higher among patients with a combination regimen versus a monotherapy regimen at index $(14.0 \%$ versus $10.1 \%)$. The probability of augmentation increased to $16.9 \%$ overall during the 5-year study period. Patients on a combination regimen remained more likely to augment therapy than patients with a monotherapy regimen (29.4\% versus $15.7 \%)$.
The probability of switching the index regimen 1-year following the index date was $6.6 \%$ (Fig. 6). This percentage was slightly higher among patients with index combination therapy than patients with index monotherapy $(10.5 \%$ versus $6.2 \%)$. The probability of switching increased to $10.0 \%$ overall during the 5 -year study period. Patients on a combination

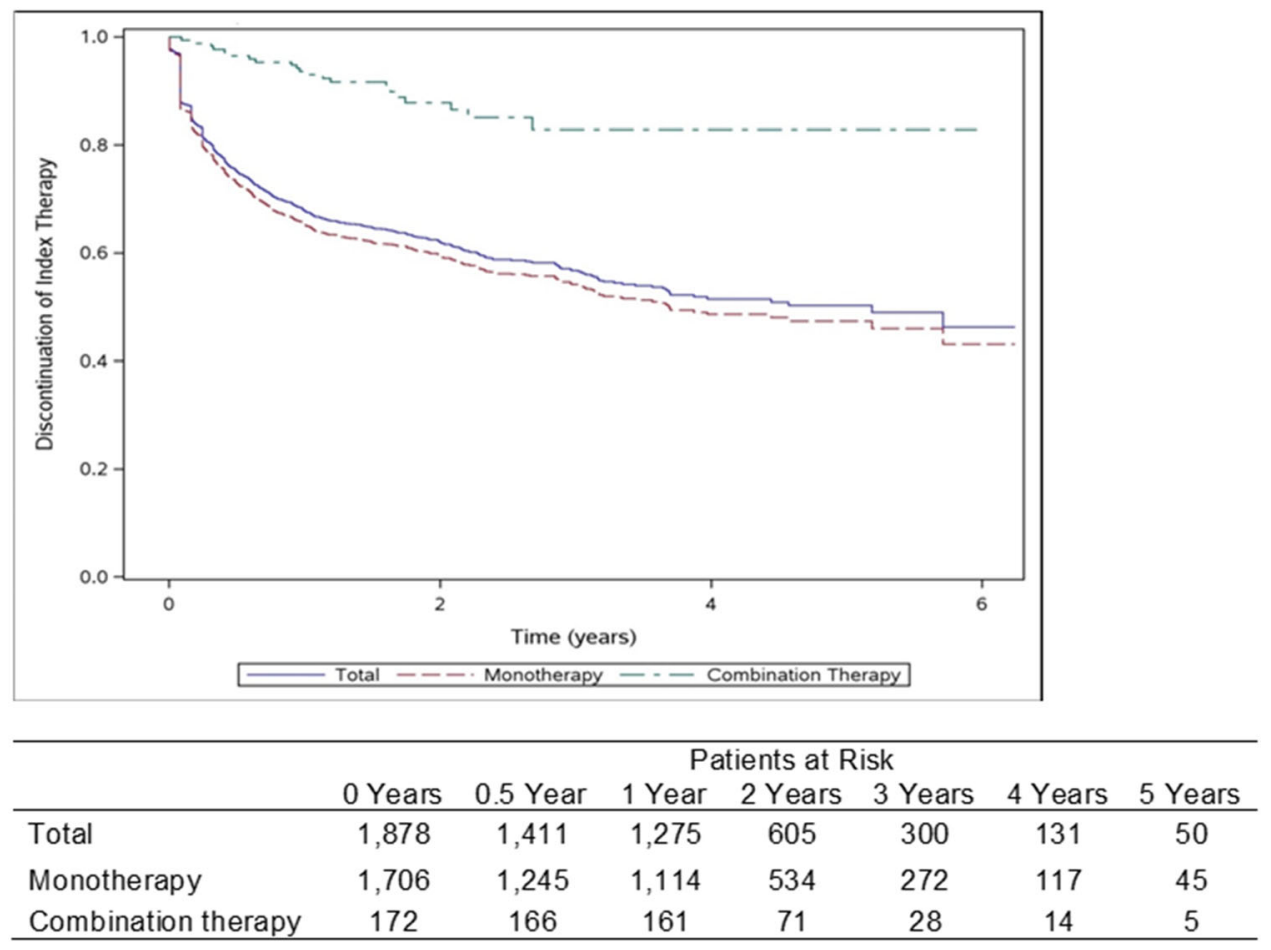

Fig. 4 Kaplan-Meier analysis of time to discontinuation 


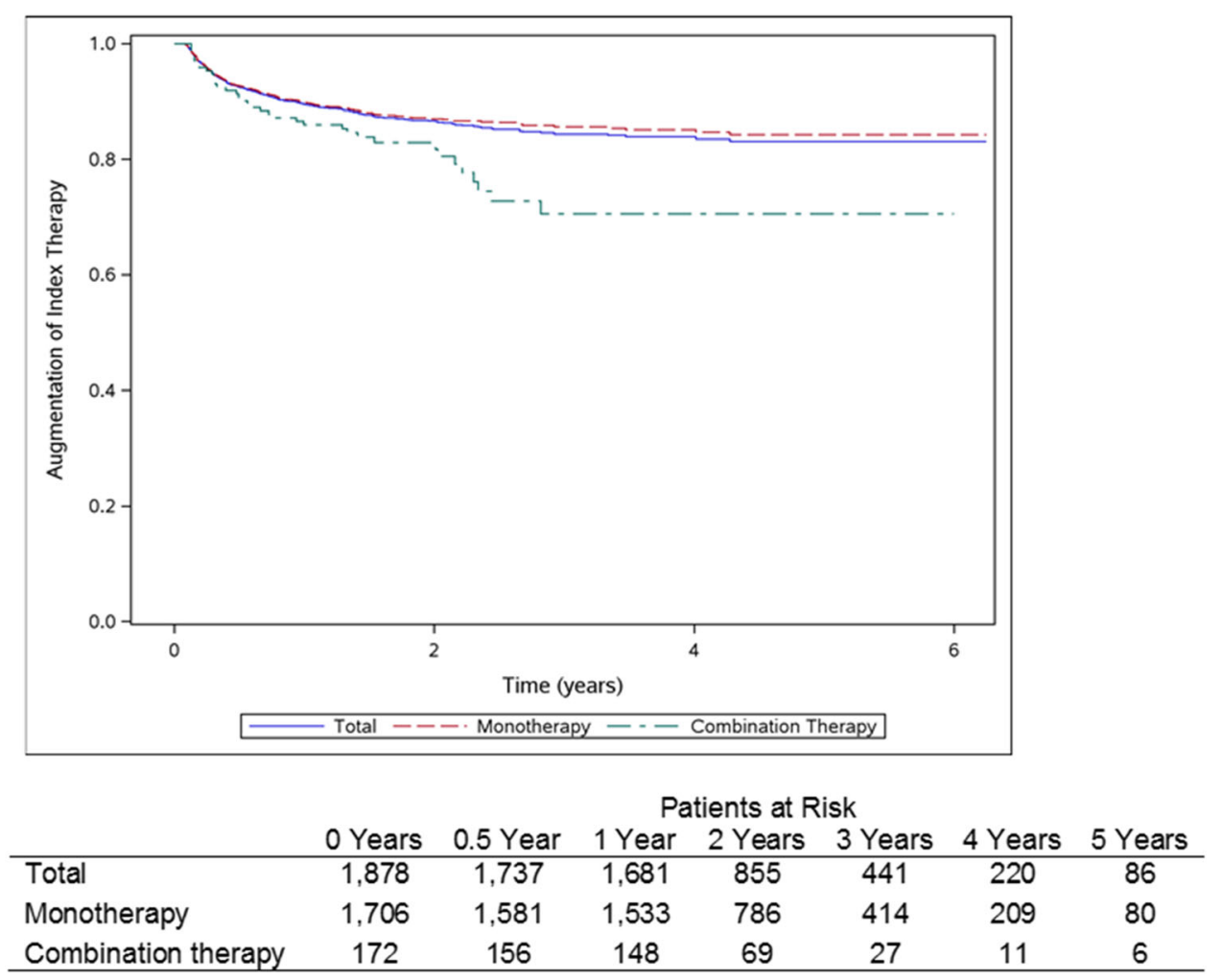

Fig. 5 Kaplan-Meier analysis of time to augmentation

regimen remained more likely to switch than patients on a monotherapy regimen $(23.4 \%$ versus $8.7 \%$ ).

Of patients who modified or discontinued their index regimen $(n=1168), 78.4 \%$ did so within 1 year of the index date. The months to therapy discontinuation or modification decreased from $10.3 \pm 9.9$ months in 2012-2013 to $9.4 \pm 9.5$ months in 2014-July 2015 to $4.9 \pm 5.2$ months in August 2015-March 2017. Patients on an index combination regimen had higher persistence than patients on an index monotherapy regimen (11.6 \pm 10.3 months versus $7.8 \pm 9.9$ months). Patients prescribed an ERA had higher persistence than patients prescribed a PDE5i ( $9.4 \pm 10.2$ versus $8.5 \pm 10.2$ months).

Almost one-third of patients (30.2\%) had a second treatment regimen (Fig. 2b). Among patients with a second regimen, $15.9 \%$ resumed their index treatment after an earlier discontinuation, all of which were on monotherapy (more than $90 \%$ were on a PDE5i).
Approximately half $(50.4 \%)$ of patients had combination therapy as their second regimen. The percentage of patients who initiated dual therapy as a second regimen remained stable over the time periods; however, the proportion of patients who initiated triple therapy as a second regimen increased from $4.0 \%$ in 2012-2013 to $12.3 \%$ in August 2015-March 2017. Patients were most commonly prescribed an ERA plus PDE5i across all time periods ranging from $57.0 \%$ of second combination regimens in 2012-2013 to $46.0 \%$ of all second combination regimens in August 2015-March 2017 (data not shown). Approximately $8.1 \%$ of patients were prescribed triple therapy as their second regimen, usually an ERA, PDE5i, and prostacyclin $(87.0 \%)$, of which $17.4 \%$ had monotherapy as their index regimen.

Over the course of the study, $24.9 \%$ of all patients initiated combination therapy, including as an index regimen. The percentage of patients with an index combination regimen increased steadily over the time periods; 


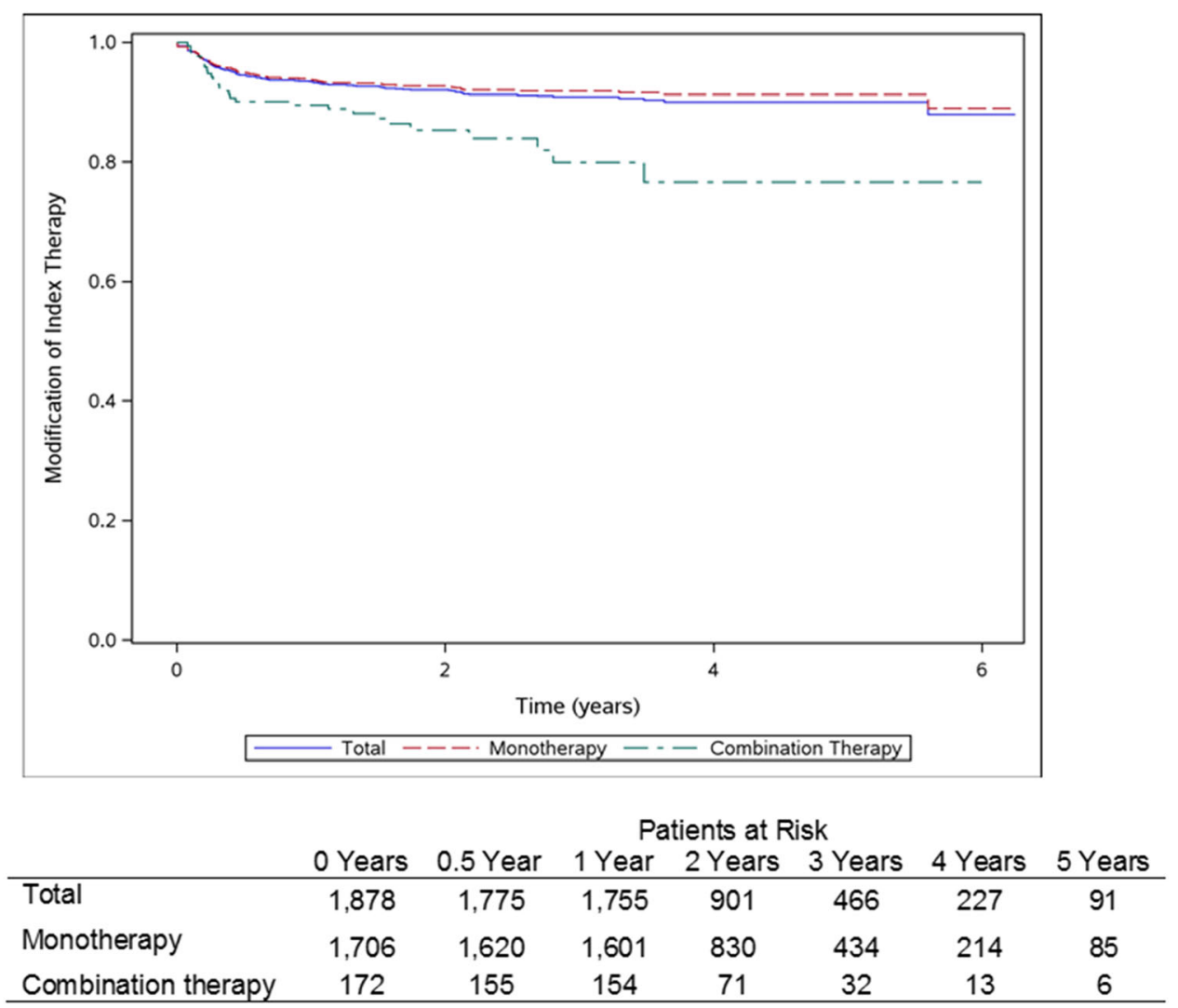

Fig. 6 Kaplan-Meier analysis of time to modification

however, the percentage of patients who had combination therapy at any time during the study was similar between the time periods (23.1\% in 2012-2013, 25.0\% in 2014-July 2015, and $26.4 \%$ in August 2015-March 2017). The mean time to combination therapy initiation was 6.5 months overall (including the 172 patients whose index regimen consisted of combination therapy); however, the mean decreased from 10.5 months in 2012-2013 to 6.4 months in 2014-July 2015 to 3.4 months in August 2015-March 2017. Among patients who initiated on monotherapy and later started combination therapy $(n=295 / 1706), \quad 78.3 \%$ began combination therapy as the second regimen and $21.7 \%$ started in the third or later regimens. Patients who transitioned to combination therapy from index monotherapy with either an ERA or PDE5i were most likely to have an ERA plus PDE5i combination regimen (data not shown). Those who initiated prostacyclin monotherapy and transitioned to combination therapy were most likely to transition to a regimen of a PDE5i plus a prostacyclin. Approximately $67.2 \%$ of patients who initiated combination therapy after index monotherapy did so within the first 6 months and $70.5 \%$ of patients did so within 1 year of the index date. Among patients who remained on any $\mathrm{PAH}$ medication until the end of the study period, $29.3 \%(n=374 / 1277)$ were on combination therapy, either remaining on their index combination therapy until the end of the study period or initiating combination therapy in subsequent regimens. Of these, $13.6 \%$ of patients were on a triple therapy regimen $(n=51 / 374)$.

\section{DISCUSSION}

This study examined real-world treatment patterns at varying time points prior to and following publication of the 2015 ESC/ERS 
treatment guidelines for $\mathrm{PAH}$. This guideline update offered physicians the option to treat patients with low- and moderate-risk PAH either upfront or sequentially with combination therapy. Most patients in this study (90.8\%) initiated a monotherapy regimen as their index regimen with almost two-thirds of all study participants initiating PDE5i monotherapy and one-sixth initiating an ERA.

The percentage of patients with upfront combination therapy increased from $5.7 \%$ in $2012-2013$ to $13.0 \%$ following the publication of updated ESC/ERS guidelines. These percentages are much lower than the $45 \%$ on combination therapy in the REVEAL registry [26] and similar to proportions receiving combination therapy in previous claims studies, which ranged from 5\% to 20\% [27-30]. Patients in the REVEAL registry were not newly diagnosed or new initiators of treatment (more than half of patients were functional class III or IV and were referred to PAH specialty centers), which likely explains the high percentage of patients on combination therapy. In our study, the increase in patients initiating treatment of $\mathrm{PAH}$ with combination therapy in the later years likely indicates the impact of recent research suggesting increased benefits to patients who initiate with combination therapy and guideline recommendations for earlier use. As a result of limited evidence from long-term clinical trials on the best combination regimens, the combination of tadalafil plus ambrisentan is the only class I recommendation for initial combination therapy; however, macitentan plus sildenafil, riociguat plus bosentan, selexipag plus an ERA or PDE5i, and sildenafil plus epoprostenol received class I evidence in support of sequential combination therapy. The percentage of patients who initiated a regimen of an ERA plus PDE5i increased from $48.7 \%$ in $2012-2013$ to $70.4 \%$ in August 2015-March 2017, whereas the percentage of patients initiating a PDE5i plus prostacyclin decreased from $35.1 \%$ to $12.5 \%$ during the same time periods. This suggests that physicians were prescribing according to guideline recommendations.

A quarter of study patients used combination therapy over the study period with the time to sequential combination therapy decreasing from 10.5 months in $2012-2013$ to 3.6 months in August 2015-March 2017. This finding supports the guideline recommendation of intensification of therapy until an adequate clinical response is observed. Given the progressive nature of $\mathrm{PAH}$, monotherapy may not be enough to elicit the response needed for many patients.

Upfront combination therapy was associated with greater adherence, persistence, and lower rates of discontinuation. Only $36.5 \%$ of index monotherapy patients remained on the index regimen without a discontinuation or modification until the end of the study, whereas $51.2 \%$ of index combination therapy users did so. Patients on combination therapy may have had better clinical responses to treatment, thus remaining on their medication longer. Adherence, as measured by PDC, was more than $10 \%$ higher for combination therapy users than for those on monotherapy. On the basis of the results of this study, physicians may consider starting or escalating combination therapy earlier to maximize treatment adherence and persistence.

\section{Limitations}

Results of this claims database analysis must be interpreted in light of inherent limitations. ICD-9-CM and ICD-10-CM codes used for patient identification were for pulmonary hypertension, not PAH specifically; thus, patients may have been misidentified, particularly those with group 2 or 3 pulmonary hypertension. To reduce the likelihood of misidentification, we required both NDC pharmacy codes and ICD-9-CM/ICD-10-CM codes for patient identification. Pulmonary hypertension is often misdiagnosed and/or treated inappropriately, potentially as $\mathrm{PAH}$, particularly if the diagnosis is not made within a specialty center and it is possible that patients with group 2/3 PH were inadvertently included. Evidence of a dispensed medication does not indicate that the medication was consumed or taken as prescribed. Additionally, medications provided as samples by the physician or as part of a clinical trial may not be accounted for in claims data. 
Lastly, this study was conducted in a large US managed care population and may not be generalizable to other populations or all patients with a PAH diagnosis.

\section{CONCLUSIONS}

The majority of patients initiated monotherapy treatment for PAH, most often PDE5is, despite market availability of several new medications. Patients with upfront combination therapy increased and the time to sequential combination therapy decreased following publication of the 2015 ESC/ERS guidelines, indicating that physicians were prescribing according to recommended guidelines. Patients who initiated combination therapy had greater adherence and persistence with fewer discontinuations than patients who initiated monotherapy, but further research is needed to confirm these results.

\section{ACKNOWLEDGEMENTS}

Funding. Actelion Pharmaceuticals US, Inc. also funded the journal's Rapid Service.

Medical Writing and/or Editorial Assistance. Deja Scott-Shemon, Optum, provided medical writing services, which was funded by Actelion.

Authorship. All named authors meet the International Committee of Medical Journal Editors (ICMJE) criteria for authorship for this article, take responsibility for the integrity of the work as a whole, and have given their approval for this version to be published.

Disclosures. Janis Pruett is an employee of Actelion Pharmaceuticals US, Inc., which funded this study. Yuen Tsang is an employee of Actelion Pharmaceuticals US, Inc., which funded this study. William Drake is an employee of Actelion Pharmaceuticals US, Inc., which funded this study. Sean Studer provided consulting services to Actelion. Michael Hull is an employee of Optum, which was contracted by Actelion to conduct this study. Caitlin Elliott is an employee of Optum, which was contracted by Actelion to conduct this study.

Compliance with Ethics Guidelines. This study did not require the use of identifiable protected health information; thus, institutional board approval or waiver of authorization was not required.

Data Availability. The data contained in our database contains proprietary elements owned by Optum and, therefore, cannot be broadly disclosed or made publicly available at this time. The disclosure of this data to thirdparty clients assumes certain data security and privacy protocols are in place and that the thirdparty client has executed our standard license agreement which includes restrictive covenants governing the use of the data.

Open Access. This article is distributed under the terms of the Creative Commons Attribution-NonCommercial 4.0 International License (http://creativecommons.org/licenses/ by-nc/4.0/), which permits any non-commercial use, distribution, and reproduction in any medium, provided you give appropriate credit to the original author(s) and the source, provide a link to the Creative Commons license, and indicate if changes were made.

\section{REFERENCES}

1. Peacock AJ, Murphy NF, McMurray JJ, Caballero L, Stewart S. An epidemiological study of pulmonary arterial hypertension. Eur Respir J. 2007;30:104-9.

2. Frost AE, Badesch DB, Barst RJ, et al. The changing picture of patients with pulmonary arterial hypertension in the United States: how REVEAL differs from historic and non-US contemporary registries. Chest. 2011;139:128-37.

3. Fox BD, Shtraichman O, Langleben D, Shimony A, Kramer MR. Combination therapy for pulmonary arterial hypertension: a systematic review and meta-analysis. Can J Cardiol. 2016;32:1520-30. 
4. Lajoie AC, Lauziere G, Lega JC, et al. Combination therapy versus monotherapy for pulmonary arterial hypertension: a meta-analysis. Lancet Respir Med. 2016;4:291-305.

5. McLaughlin VV, Oudiz RJ, Frost A, et al. Randomized study of adding inhaled iloprost to existing bosentan in pulmonary arterial hypertension. Am J Respir Crit Care Med. 2006;174:1257-63.

6. Simonneau G, Rubin LJ, Galie N, et al. Addition of sildenafil to long-term intravenous epoprostenol therapy in patients with pulmonary arterial hypertension: a randomized trial. Ann Intern Med. 2008;149:521-30.

7. Galie N, Barbera JA, Frost AE, et al. Initial use of ambrisentan plus tadalafil in pulmonary arterial hypertension. N Engl J Med. 2015;373:834-44.

8. Sitbon O, Channick R, Chin KM, et al. Selexipag for the treatment of pulmonary arterial hypertension. N Engl J Med. 2015;373:2522-33.

9. Jansa P, Pulido T. Macitentan in pulmonary arterial hypertension: a focus on combination therapy in the SERAPHIN trial. Am J Cardiovasc Drugs. 2018;18:1-11.

10. Pulido T, Adzerikho I, Channick RN, et al. Macitentan and morbidity and mortality in pulmonary arterial hypertension. N Engl J Med. 2013;369: 809-18.

11. Channick RN, Delcroix M, Ghofrani HA, et al. Effect of macitentan on hospitalizations: results from the SERAPHIN trial. JACC Heart Fail. 2015;3: $1-8$.

12. Galie N, Humbert M, Vachiery JL, et al. 2015 ESC/ ERS guidelines for the diagnosis and treatment of pulmonary hypertension: the Joint Task Force for the Diagnosis and Treatment of Pulmonary Hypertension of the European Society of Cardiology (ESC) and the European Respiratory Society (ERS): Endorsed by: Association for European Paediatric and Congenital Cardiology (AEPC), International Society for Heart and Lung Transplantation (ISHLT). Eur Heart J. 2016;37:67-119.

13. Galie N, Channick RN, Frantz RP, et al. Risk stratification and medical therapy of pulmonary arterial hypertension. Eur Respir J. 2019;53:1801889.

14. Austin ED, Kawut SM, Gladwin MT, Abman SH. Pulmonary hypertension: NHLBI workshop on the primary prevention of chronic lung diseases. Ann Am Thorac Soc. 2014;11(Suppl 3):S178-85.

15. Ryan JJ, Butrous G, Maron BA. The heterogeneity of clinical practice patterns among an international cohort of pulmonary arterial hypertension experts. Pulm Circ. 2014;4:441-51.

16. Deano RC, Glassner-Kolmin C, Rubenfire M, et al. Referral of patients with pulmonary hypertension diagnoses to tertiary pulmonary hypertension centers: the multicenter RePHerral study. JAMA Intern Med. 2013;173:887-93.

17. McLaughlin VV, Langer A, Tan M, et al. Contemporary trends in the diagnosis and management of pulmonary arterial hypertension: an initiative to close the care gap. Chest. 2013;143:324-32.

18. Farber HW, Miller DP, Meltzer LA, McGoon MD. Treatment of patients with pulmonary arterial hypertension at the time of death or deterioration to functional class IV: insights from the REVEAL registry. J Heart Lung Transplant. 2013;32:1114-22.

19. McLaughlin VV, Archer SL, Badesch DB, et al. ACCF/AHA 2009 expert consensus document on pulmonary hypertension a report of the American College of Cardiology Foundation Task Force on Expert Consensus Documents and the American Heart Association developed in collaboration with the American College of Chest Physicians; American Thoracic Society, Inc.; and the Pulmonary Hypertension Association. J Am Coll Cardiol. 2009;53:1573-619.

20. Studer S, Hull M, Pruett J, Koep E, Tsang Y, Drake W 3rd. Treatment patterns, healthcare resource utilization, and healthcare costs among patients with pulmonary arterial hypertension in a real-world US database. Pulm Circ. 2019;9:2045894018816294.

21. Quan H, Li B, Couris CM, et al. Updating and validating the Charlson comorbidity index and score for risk adjustment in hospital discharge abstracts using data from 6 countries. Am J Epidemiol. 2011;173:676-82.

22. Bayliss EA, Ellis JL, Shoup JA, Zeng C, McQuillan DB, Steiner JF. Association of patient-centered outcomes with patient-reported and ICD-9-based morbidity measures. Ann Fam Med. 2012;10: 126-33.

23. Clinical Classification Software (CCS) for ICD-9CM. Agency for healthcare research and quality, Rockville, MD. http://www.hcup-us.ahrq.gov/ toolssoftware/ccs/ccs.jsp. Comorbidities defined by Healthcare Cost and Utilization Project (HCUP) comorbidity software. 2015. Agency for healthcare research and quality (AHRQ), Rockville, MD. www. hcup-us.ahrq.gov/toolssoftware/comorbidity/como rbidity.jsp. Accessed Sept 2018.

24. Nau D. Proportion of days covered (PDC) as a preferred method of measuring medication adherence. 
Springfield: Pharmacy Quality Alliance; 2012. p. 2012.

25. Basak R, McCaffrey Iii DJ, Bentley JP, Przybyla SM, West-Strum D, Banahan BF. Adherence to multiple medications prescribed for a chronic disease: a methodological investigation. J Manag Care Spec Pharm. 2014;20:815-23.

26. McGoon MD, Miller DP. REVEAL: a contemporary US pulmonary arterial hypertension registry. Eur Respir Rev. 2012;21:8-18.

27. Burger CD, Ozbay AB, Lazarus HM, et al. Treatment patterns and associated health care costs before and after treatment initiation among pulmonary arterial hypertension patients in the United States. J Manag Care Spec Pharm. 2018;24:1-9.
28. Copher R, Cerulli A, Watkins A, Laura Monsalvo M. Treatment patterns and healthcare system burden of managed care patients with suspected pulmonary arterial hypertension in the United States. J Med Econ. 2012;15:947-55.

29. Angalakuditi M, Edgell E, Beardsworth A, Buysman E, Bancroft T. Treatment patterns and resource utilization and costs among patients with pulmonary arterial hypertension in the United States. J Med Econ. 2010;13:393-402.

30. Sikirica M, Iorga SR, Bancroft T, Potash J. The economic burden of pulmonary arterial hypertension (PAH) in the US on payers and patients. BMC Health Serv Res. 2014;14:676. 